Pacific

Journal of

Mathematics

\title{
GROUP ACTIONS ON POLYNOMIAL AND POWER SERIES RINGS
}

Peter Symonds 


\title{
GROUP ACTIONS ON POLYNOMIAL AND POWER SERIES RINGS
}

\author{
PETER Symonds
}

\begin{abstract}
When a finite group $G$ acts faithfully on a graded integral domain $S$ which is an algebra over a field $k$, such as a polynomial ring, we consider $S$ as a $k G$-module. We show that $S$ is asymptotically mostly projective in each degree, and also that it is in fact mostly free in an appropriate sense. Similar results also hold for filtered algebras, such as power series rings.
\end{abstract}

\section{Introduction.}

Let $S=\bigoplus_{n=0}^{\infty} S_{n}$ be a graded algebra over a field $k$. We suppose that $S$ is finitely generated over $k$ as a $k$-algebra and that the homogeneous components $S_{n}$ are finite dimensional vector spaces over $k$. Let $G$ be a finite group of grading preserving automorphisms of $S$ (so $G$ acts faithfully). We are concerned with the structure of $S$ as a $k G$-module.

The classical theory of Hilbert and Serre asserts that for large $n, \operatorname{dim}_{k} S_{n}$ is given by a function

$$
\phi_{S}(n)=c_{d-1}(n) n^{d-1}+c_{d-2}(n) n^{d-2}+\cdots+c_{0}(n),
$$

where the $c_{i}(n)$ are rational valued functions periodic in $n$, i.e., $\phi_{i}(n+$ $p)=\phi_{i}(n)$ for some integer $p$ (see Section 2). If $c_{d-1}$ is assumed not to be identically zero then $d$ is equal to the dimension of the ring in various senses. If $S$ is a polynomial ring then $d$ is equal to the number of variables.

From now on, we assume that $S$ is an integral domain. Let $P_{n}$ denote the maximal projective summand of $S_{n}$ (defined up to isomorphism).

Theorem 1.1. $\operatorname{dim}_{k}\left(S_{n} / P_{n}\right)$ is bounded by a polynomial in $n$ of degree $d-2$.

Thus $S_{n}$ is mostly projective, and if $S$ is a polynomial ring then the non-projective part grows like a polynomial ring in one fewer variables.

In fact $S$ is mostly free, although the individual $S_{n}$ do not have to contain a free module at all; the different projectives can occur in different degrees. To explain this let $R=S^{G}$, the ring of invariants. 
Theorem 1.2. $S$ contains a free $k G$-submodule $F$ of rank 1 , a sum of homogeneous pieces, such that the product map $R \otimes_{k} F \rightarrow S$ is injective. Denote its image by $R F=\bigoplus_{n}(R F)_{n}$. Then $R F$ is a free summand of $S$ and $\operatorname{dim}_{k}\left(S_{n} /(R F)_{n}\right)$ is bounded by a polynomial of degree $d-2$.

Of course, the first theorem is a corollary to the second. Versions of these theorems were proven by Howe [4] in characteristic 0 and by Bryant $[\mathbf{2}, \mathbf{3}]$ for polynomial rings.

Section 2 contains the main proof, except for some technical details which appear in Section 3. Section 4 proves similar results for filtered algebras.

\section{Main Proof.}

Proof. We can assume that $k$ is a splitting field for $G$, since if a $k G$-module contains a free or projective summand after extension of scalars then it did so before. Let $Q_{S}$ (resp. $Q_{R}$ ) denote the fields of fractions of $S$ (resp. $R$ ), so $Q_{S} \cong Q_{R} \otimes_{R} S$. By the Normal Basis Theorem, $Q_{S}$ is a free module of rank 1 over $Q_{R} G$; let $e$ be a generator. Then, over $k G$, $e$ generates a free submodule $E$ of rank 1 such that $Q_{S} \cong Q_{R} \otimes_{k} E$. Now there is an $r \in R$ such that $r e \in S$. Let $F$ be the $k G$-module generated by re, so $F \subset S$ and $F=r E \cong k G$. Also the product map $R \otimes_{k} F \rightarrow R F \subset S$ is injective.

We claim that $F$ can be assumed to be a sum of homogeneous pieces, $F=\bigoplus_{i} F_{n_{i}}$. The proof of this plausible statement is somewhat delicate, and we postpone it to the next section.

Let $x_{1}, \ldots, x_{s}$ be homogeneous generators for $S$ as a $k$-algebra. Then $x_{i}=\sum_{j} \frac{a_{i j}}{b_{i j}} e_{j}$, where $a_{i, j}, b_{i, j} \in R$ and the $e_{j}$ form a homogeneous $k$-basis for $F$. By writing $b_{i, j} x_{i}=\sum_{j} a_{i, j} e_{j}$ and taking the homogeneous component of this equation in some degree where $b_{i, j} x_{i}$ is non-zero, we see that we may assume that the $b_{i, j}$ are homogeneous. Let $\alpha \in R_{a}$ be the product of all the $b_{i, j}$. Then each $x_{i} \in \alpha^{-1} R F$, so $S \subset \alpha^{-1} R F$.

Thus

$$
(R F)_{n} \subset S_{n} \subset \alpha^{-1}(R F)_{n+a},
$$

and so, identifying $R F$ with $R \otimes_{k} F$, we have

$$
\bigoplus_{i} R_{n-n_{i}} \otimes F_{n_{i}} \subset S_{n} \subset \alpha^{-1} \bigoplus_{i} R_{n+a-n_{i}} \otimes F_{n_{i}} .
$$

In particular, the dimension of $S_{n} /(R F)_{n}$ is bounded by the difference in the dimensions of the two sides, i.e., by

$$
\sum_{i}\left(\phi_{R}\left(n+a-n_{i}\right)-\phi_{R}\left(n-n_{i}\right)\right) \operatorname{dim}_{k} F_{n_{i}} .
$$


But

$$
\begin{aligned}
\phi_{R}\left(n+a-n_{i}\right)-\phi_{R}( & \left.-n_{i}\right)=c_{d-1}\left(n+a-n_{i}\right)\left(n+a-n_{i}\right)^{d-1} \\
& -c_{d-1}\left(n-n_{i}\right)\left(n-n_{i}\right)^{d-1}+\text { lower degree terms, }
\end{aligned}
$$

and $c_{d-1}$ is periodic, with period dividing a (see 3.1 ), so the $n^{d-1}$ term cancels and we are done.

\section{Technical Details.}

The form of $\phi_{S}(n)$ given above is not quite the standard one, although it is quoted in [4]. The usual references deal with a module over a polynomial ring which has all the variables in degree 1 , and then all the coefficients of $\phi$ are constants. To deduce the version given in the introduction, note that if $S$ is generated by $x_{1}, \ldots, x_{s}$ then it is a finitely generated module over $k\left[x_{1}, \ldots, x_{s}\right]$. By taking suitable powers $y_{i}$ of the $x_{i}$ we can get all the $y_{i}$ in the same degree $b$, and $S$ will still be finitely generated over $k\left[y_{1}, \ldots, y_{s}\right]$. For $0 \leq j \leq b-1$, set $T_{j}=\bigoplus_{l=0}^{\infty} S_{j+l b}$. Then $R \cong \bigoplus_{j} T_{j}$ as a $k\left[y_{1}, \ldots, y_{s}\right]-$ module, and after regrading each $T_{j}$ so that each $y_{i}$ can have degree 1 , we can apply the usual theory ([1] 11.2, [5] VII Theorem 41) to each $T_{j}$ and sum the results. It is the summation that leads to the periodic coefficients.

Lemma 3.1 ([4]). If $R$ is an integral domain (as it always is for us), $c=$ $\operatorname{gcd}\left\{r \in \mathbb{Z} \mid R_{r} \neq 0\right\}$ and $\phi_{R}(n)=c_{d-1}(n) n^{d-1}+\cdots+c_{0}(n)$, then there is a constant $b$ such that

$$
c_{d-1}(n)= \begin{cases}b, & \text { if } n \mid c, \\ 0, & \text { otherwise. }\end{cases}
$$

Proof. If $0 \neq \alpha \in R_{a}$, then multiplication by $\alpha$ embeds $R_{n}$ in $R_{n+a}$, so for large $n, \phi_{R}(n) \leq \phi_{R}(n+a)$. Now consider the limit of $\phi_{R}(n) / n^{d-1}$ as $n \rightarrow \infty$ through elements of the same residue class modulo the period of $c_{d-1}$ to see that $c_{d-1}(n) \leq c_{d-1}(n+a)$. This, together with the periodicity, implies the result.

Now we prove the claim made in Section 2.

Proposition 3.2. The free module $F \subset S$ can be assumed to be a sum of homogeneous pieces in such a way that the product map $Q_{R} \otimes_{k} F \rightarrow Q_{S}$ is still an isomorphism.

Proof. For each simple $k G$-module $V$, let $T_{V}=\operatorname{Hom}_{k G}(V, S)$, a graded $R$ module. Now $\operatorname{soc} F$ is a direct sum of simples. Let $\operatorname{soc}_{V}(F)$ denote the sum

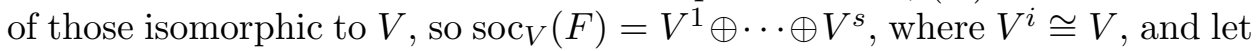
$P_{V^{i}}$ be a projective summand of $F$ with $\operatorname{soc}\left(P_{V^{i}}\right)=V^{i}$. The inclusions of the $V^{i}$ in $S$ give us $s$ homomorphisms $f^{i} \in T_{V}$, which are linearly independent over $R$. 
Lemma 3.3. Let $f^{1}, \ldots, f^{s}$ be elements of a graded $R$-module $T$ which are linearly independent over $R$. Write each $f^{j}$ as a sum of its homogeneous components; $f^{j}=\sum_{k} f_{k}^{j}, f_{k}^{j} \in T_{k}$. Then for each $j$ there is an integer $k_{j}$ such that $f_{k_{1}}^{1}, \ldots, f_{k_{s}}^{s}$ are linearly independent over $R$.

Proof. For each $0 \leq t \leq s$, let $P_{t}$ be the claim that there exist integers $k_{1}, \ldots, k_{t}$ such that $f_{k_{1}}^{1}, \ldots, f_{k_{t}}^{t}, f^{t+1}, \ldots, f^{s}$ are linearly independent over $R$. $P_{0}$ is true by hypothesis and we want $P_{s}$. We give a proof by induction on $t$, so assume $P_{t}$.

If $P_{t+1}$ is false, then for each $k \in \mathbb{Z}$ we can find $u_{k}, r_{k}^{i} \in R, u_{k} \neq 0$, such that

$$
u_{k} f_{k}^{t+1}=r_{k}^{1} f_{k_{1}}^{1}+\cdots+r_{k}^{t} f_{k_{t}}^{t}+r_{k}^{t+2} f^{t+2}+\cdots+r_{k}^{s} f^{s} .
$$

Let $u$ be the product of the $u_{k}$ for which $f_{k}^{t+1} \neq 0$. Then $u f^{t+1}=$ $\sum_{k}\left(\frac{u}{u_{k}}\right) u_{k} f_{k}^{t+1}$, contradicting $P_{t}$.

Applying this to the $\left\{f^{i}\right\} \subset T_{V}$ we obtain homogeneous $\left\{\bar{f}^{i}\right\} \subset T_{V}, \bar{f}^{i} \in$ $T_{a_{i}}$, say, linearly independent over $R$.

Lemma 3.4. The evaluation map ev $: T_{V} \otimes_{k} V \rightarrow S$ is injective.

Proof. In fact ev : $\operatorname{Hom}_{k G}(V, M) \otimes_{k} V \rightarrow M$ is injective for any $k G$-module $M$. This is because it factors through $\operatorname{soc}_{V}(M)$, which is a direct sum of $V$ 's, so we are reduced to proving the case $M=V$. But then ev is an isomorphism, since $\operatorname{Hom}_{k G}(V, V) \cong k$, by the assumption that $k$ is a splitting field.

Corollary. The product map $R \otimes_{k}\left(\bigoplus_{i} \bar{f}^{i}(V)\right) \rightarrow S$ is injective.

Now let $\bar{P}_{V^{i}}$ be the image of the projection of $P_{V^{i}}$ to $S_{a_{i}}$. The projection map is injective on $\operatorname{soc}\left(P_{V}^{i}\right)$, by the construction of $a_{i}$, so $\bar{P}_{V^{i}} \cong P_{V^{i}}$ and $\operatorname{soc}\left(\bar{P}_{V^{i}}\right)=\bar{f}^{i}(V)$. Let $\bar{P}_{V}=\bigoplus_{i} \bar{P}_{V^{i}}$ and consider the product map $R \otimes_{k}$ $\bar{P}_{V} \rightarrow S$. Since $\operatorname{soc}\left(R \otimes_{k} \bar{P}_{V}\right)=R \otimes \bigoplus_{i} \bar{f}^{i}(V)$, it is injective on the socle, so is injective.

Finally, we sum the $\bar{P}_{V}$ over the simples $V$ to obtain $\bar{F}$, a free $k G$-module of rank 1 , which is a sum of homogeneous pieces, as required.

Remark. If $G$ is a $p$-group, where $p$ is the characteristic of $k$, then the proof is much simpler because $\operatorname{soc}(F) \cong k$. Under at least one of the projections of $F$ onto its homogeneous components the image of $\operatorname{soc}(F)$ must be nonzero. Let $\bar{F}$ be the image of $F$ under this projection. Then $\bar{F} \cong k G$ and $Q_{R} \otimes_{k} F \rightarrow Q_{S}$ is an isomorphism because it is injective on the socle, and both sides have the same dimension over $Q_{R}$.

This is enough to prove 1.1 for general $G$. For if $P=\operatorname{Syl}_{p}(G)$ then $S$ is a direct summand of $\operatorname{Ind}_{P}^{G} \operatorname{Res}_{P}^{G} S$. 
Remark. It is not hard to see that, given any degree $m$, the summands of $\bar{F}$ can be moved by multiplication by a scalar to lie in $T_{m+l c}=S_{m+l c} \oplus \cdots \oplus$ $S_{m+(l+1) c-1}$, for some $l$. The argument of the proof of 1.2 now shows that the non-free part of $T_{n}$ has dimension bounded by a polynomial of degree $d-2$ (cf. $[2,3])$.

\section{Filtered Rings.}

The case of filtered rings is slightly different. Consider the power series ring $k[[x]]$ in characteristic 2 and let the group of order 2 act by $x \mapsto x /(x+1)=$ $x+x^{2}+x^{3}+\cdots$. The action on the associated graded ring is trivial, yet the action on $k[[x]]$ certainly contains free summands (the only alternative is trivial).

We consider finitely generated $k$-algebras $S$ which are integral domains and have a filtration $S=I_{0} \subset I_{1} \subset I_{2} \subset \cdots$. Each $S / I_{n}$ is assumed to be finite dimensional over $k$, and $\cap I_{n}=\{0\}$. There is a finite group $G$ of automorphisms of $S$, which preserves the filtration. The invariants are $R=S^{G}$ with the induced filtration $J_{n}=R \cap I_{n}$. Again there is a function

$$
\chi_{S}(n)=c_{d}(n) n^{d}+c_{d-1} n^{d-1}+\cdots c_{0}(n),
$$

where the $c_{i}$ are periodic, such that $\operatorname{dim}_{k}\left(S / I_{n}\right)=\chi_{S}(n)$ for large $n$. If $S$ is a power series ring, then $d$ is equal to the number of variables.

As before there is a free $k G$-module of rank 1 in $S$, and the product map $R \otimes_{k} F \rightarrow S$ is injective. Since $F$ is finite dimensional there is some integer $f$ such that $F \cap I_{f}=0$, so $F$ injects into $S / I_{f}$.

For each $n$, let $K_{n}$ be a vector space complement to $J_{n}$ in $R$. Then the product map $K_{n} \otimes F \rightarrow S / I_{f+n}$ is injective, so its image, $K_{n} F$ is a free summand of $S / I_{f+n}$.

Proceeding in the same way as before we can prove:

Theorem 4.1. $\operatorname{dim}_{k}\left(\left(S / I_{f+n}\right) / K_{n} F\right)$ is bounded by a polynomial of degree $d-1$.

So $S$ is mostly free. Again, for a power series ring, the non-free part grows like a power series ring in one fewer variables.

\section{References}

[1] M.F. Atiyah and I.G. Macdonald, Introduction to Commutative Algebra, AddisonWesley, Reading, MA, 1969.

[2] R.M. Bryant, Symmetric powers of representation of finite groups, Jour. Algebra, 154 (1993), 416-436.

[3] _ Groups acting on polynomial algebras, Finite and locally finite groups. NATO Advanced Science Institutes Series C: Mathematical and Physical Sciences, 471, 327346, Kluwer Acad. Publ. Dordrecht, 1995. 
[4] R. Howe, Asymptotics of dimensions of invariants for finite groups, Jour. Algebra, 122 (1989), 374-379.

[5] O. Zariski and P. Samuel, Commutative Algebra, Vol. II, Van Nostrand, Princeton, 1960.

Received October 13, 1998.

Department of Mathematics

U.M.I.S.T.

MANCHESTER M60 1QD

ENGLAND

E-mail address: psymonds@umist.ac.uk 